\title{
Low temperature fluorescence spectra of atropisomeric perylene dyes
}

\author{
Heinz Langhals*, Jürg Fünfschilling†, Dieter Glatz† and \\ IRIS ZSCHOKKE-GRÄNACHER† \\ * Institut für Organische Chemie der Universität München, Karlstrasse 23, D-8000 München 2, Germany \\ and + Institut für Physik der Universität Basel, Klingelbergstr. 82, CH-4056 Basel, Switzerland
}

(Received 12 September 1986; in final form 21 July 1987; accepted 27 July 1987)

\begin{abstract}
The site selection spectra of highly photostable perylene fluorescent dyes at $4.2 \mathrm{~K}$ in a glassy matrix of three different organic solvents (ethanol, methyl tetrahydrofuran, n-butyl acetate) are presented. The spectra of the two isomers are identical for a given solvent and only very minute differences are detectable between different solvents. We conclude that the influence of the matrix on the fluorescence spectra is of only minor importance. The influence of the orientation in the matrix is negligible.
\end{abstract}

\section{INTRODUCTION}

The preparation of the atropic isomer perylene fluorescent dyes $1 \mathrm{a}$ and $\mathbf{1 b}$ has been reported in previous work [1]. These dyes have an extraordinarily high photostability, a fluorescence quantum yield of about unity [2] and a high solubility in organic solvents. All have very similar physical properties. In particular, their i.r. and u.v.-vis absorption and fluorescence spectra in organic solvents are identical at room temperature [1]. However, the molecular shapes of the dyes are different due to the different position of the bulky tert-butyl groups. Therefore we use the two isomers $1 \mathrm{a}$ and $1 \mathrm{~b}$ as probes to study the interactions of dye molecules with glassy media. At low temperature the site selection fluorescence spectra [3] of dyes give information about the interaction of the dye molecules with the matrices, as is e.g. studied in hole burning experiments [4].

\section{EXPERIMENTAL}

Dyes $1 \mathrm{a}$ and $1 \mathrm{~b}$ are prepared and purified according to the literature [1]. The light source for the site selection spectroscopy is an argon ion laser $(200 \mathrm{~mW})$. The light can be attenuated to reduce hole burning effects. The laserbeam is enlarged with a cylindrical lens to illuminate about $1 \mathrm{~cm}^{3}$ of the sample, which is a frozen solution in a glass tube inside a glass He dewar. In optical contact with the solution is a $1 \mathrm{~m}$ long quartz light guide. The other end of this light guide is imaged onto the $0.5 \mathrm{~m}$ monochromator entrance slit. The spectra are recorded with an optical multichannel analyser (OMA, PAR model 1254). Since only differences between spectra are of interest here, the spectra are not corrected either for the spectral response of the system nor for the slight nonlinear wavelength scale. The positions of the vibrational lines, however, are determined with high accuracy by the use of suitable calibration lamps.

\section{RESULTS AND DISCUSSION}

The site selection spectra of $1 \mathrm{a}$ and $1 \mathrm{~b}$ in the three solvents, ethanol, methyl tetrahydrofurane and n-butyl acetate are given in Fig. 1.
The line positions in the six spectra are identical for corresponding lines and also the spectral shapes of 1a and $1 \mathrm{~b}$ spectra for a given solvent are almost identical Note that those features of the spectra that depend on hole burning, especially the ratio zero-phonon line intensity/phonon-wing intensity, may easily be influenced by slight geometrical changes from experiment to experiment and are thus not significant. However, the different relative intensities of lines 1,2 and 3 in the different solvents are significant and reproducible and represent a true solvent effect.

From these findings we may conclude, that (1) the dyes $1 \mathrm{a}$ and $1 \mathrm{~b}$ are only very little influenced by the solvents and (2) despite the different geometric shape of the two isomers the spectra are identical. They are mainly governed by the structure of the dye and only very little by the global interaction with the solvent $[5,6]$.
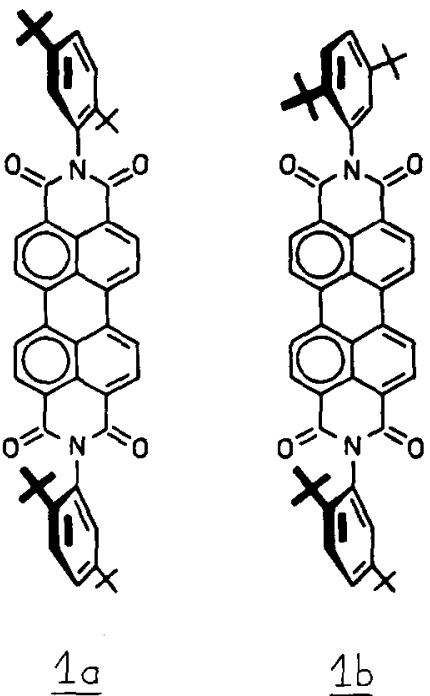

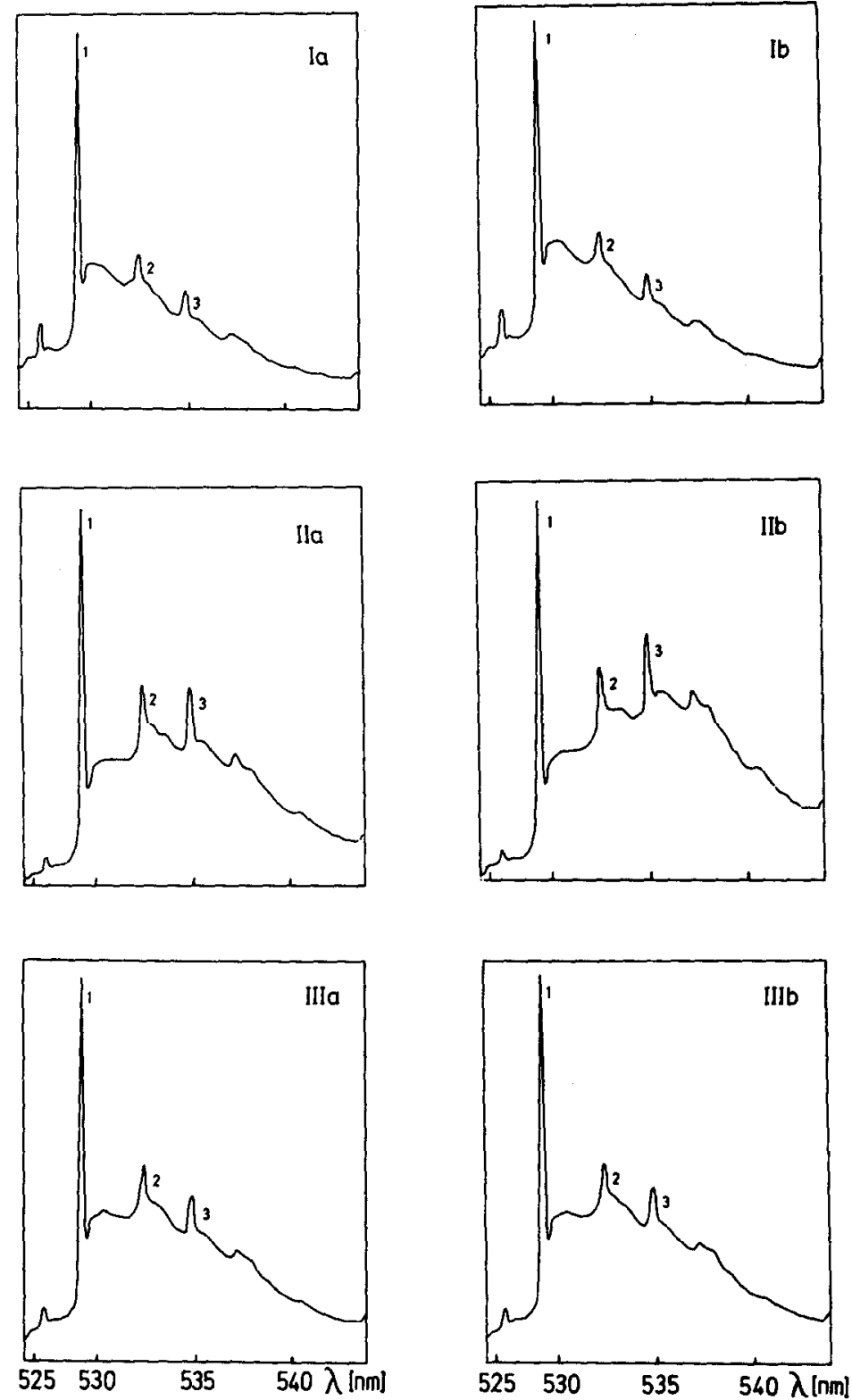

Fig. 1. Fluorescence spectra of $1 \mathrm{a}$ (left) and $1 \mathrm{~b}$ (right) in ethanol (top), methyl tetrahydrofuran (middle) and n-butyl acetate(bottom) at $4.2 \mathrm{~K}$ normalized to the height of the largest line 1 . Excitation: $514.5 \mathrm{~nm}$. The corrcsponding line positions are identical within the experimental error of $0.6 \mathrm{~cm}^{-1}$. These positions are: $18^{\prime} 892.7 \mathrm{~cm}^{-1}(1), 18^{\prime} 786.0 \mathrm{~cm}^{-1}$ (2) and $18^{\prime} 702.1 \mathrm{~cm}^{-1}$ (3).

Acknowledgement--This work was supported by the Stiftung Volkswagenwerk and the Swiss National Science Foundation.

\section{REFERENCES}

[1] H. Langhals, Chem. Ber. 118, 4641 (1985).

[2] A. Rademacher, S. Märkle and H. Langhals, Chem. Ber. 115, 2927 (1982)
[3] R. I. Personov and E. I. Al'Shits, Chem. Phys. Lett. 33 85 (1975).

[4] J. FRIEDRICH and D. HAARER, Angew. Chem. Int. Ed. Engl 23, 113 (1984)

[5] C. AUBERT, J. FÜNFSCHILling, I. ZSCHOKKEGränacher and H. Langhals, Z. Analyt. Chem. 320, 361 (1985)

[6] H. Langhals, Chem. Industrie 37, 470 (1985) 